\title{
The Universality, Major Harm and Deep Causes of Irrational Construction Periods
}

\author{
Sun Jiajun* \\ Petrochina Changqing Oilfield Company Supervision Department, Xian, China \\ *Corresponding Author: Sun Jiajun, Petrochina Changqing Oilfield Company Supervision Department, Xian, China.
}

Received: February 23, 2022; Published: February 28, 2022

DOI: 10.55162/MCET.02.023

\begin{abstract}
Rome was not built in a day, the Long March was not completed in one step, and the project period has its own objective laws. Respecting the objective law of the project duration, consciously using this law to serve the project, and acknowledging that the project duration is incompressible under the premise of various constraints are the theoretical qualities that every project manager should have. It is an extremely wrong concept to regard the project duration as a "sponge that is still empty at will be squeezed", and the irrational construction period under the guidance of this concept is a universal problem in project management.
\end{abstract}

Keywords: engineering duration irrational

\section{Give the "infrastructure maniac" an image}

In order to give an image to the "infrastructure maniac", first of all, it is necessary to give a quantitative image of China's territory, which is the five threes about the China Tower, that is, thirty-three thousand three hundred and thirty-three, which is the Arabic number string: 33333.

The penultimate "3" refers to the three strategic zones in the overall pattern of integration, which are the Yangtze River Delta, the Greater Bay Area and the Beijing-Tianjin-Hebei Region. These three strategic zones play a pivotal role in China's economic construction, are the political center, economic center and innovation center of modern China, and are the top of the China Tower.

The penultimate " 3 " refers to almost thirty provincial capitals and more than thirty provincial-level administrative units closely linked to them. Shaanxi-Gansu-Ningxia-Yunnan-Guichuan two lakes, two guang, two rivers and mountains. The cities where the administrative centers of these provincial-level administrative districts are located are directly called, that is, directly referred to. All in all, almost thirty provincial capitals and more than thirty provincial-level administrative units closely linked to them are the shoulders of the Chinese tower.

The penultimate " 3 " refers to almost three hundred prefecture-level cities. This urban agglomeration composed of prefecture-level cities is a direct manifestation of China's vastness, regional characteristics, and many ethnic members, and is the tower of the Chinese Tower.

The penultimate " 3 " refers to those county-level administrative regions on the land of China with a numerical scale of not less than 3,000 . They are the transit points for the radiation extension of modern civilization to a wider and more marginal area, the foundation of modern China's political stability, ethnic unity and economic development, and the tower base of China's tower.

The penultimate " 3 " refers to the 30,000 townships embedded in the sky like stars on the vast coastal territory along the lingnan ridge north along the yangtze river on both sides of the Great Wall. There is the nostalgia of the Chinese nation for generations, and the 
green waters and green mountains there are the golden mountains and silver mountains of modern China. It is the root of the original ecological ethnic culture and the base of the Chinese tower.

\section{A tower, its name is China}

Thirty thousand townships on the base are about the same.

\section{A tower, its name is China}

The 3000 counties and cities on the base of the tower are similar.

\section{A tower, its name is China}

The three hundred cities on the tower are about the same.

\section{A tower, its name is China}

Thirty provincial capitals on the shoulders of the tower are about the same.

\section{A tower, its name is China}

The three major strategic powers on the top of the tower can be the town.

\section{A tower, its name is China}

Needless to say, the Chinese dream on the spire.

China, which is in the process of modernization, industrialization and urbanization, is vividly called "infrastructure demon" by all countries in the world. How big are China's infrastructure projects? How to describe this "infrastructure maniac" to give a holistic impression? Let us take this "China Tower" model, which describes the vastness of China's territory, as a mathematical model. Because of the help of this model, it is easy for people to establish a holistic and quantitative image of "how big is the scale of China's infrastructure construction projects"; because with the help of this model, it is easy to establish a holistic and quantitative image of "infrastructure madness, that is, how strong China's industrialization ability in infrastructure construction is". In various documents on China's economic construction, we can always see quantitative words such as the "Eleventh Five-Year Plan", "Twelfth Five-Year Plan" and "Thirteenth Five-Year Plan", and their real meaning is "Eleventh Five-Year Plan", "Twelfth Five-Year Plan" and "Thirteenth Five-Year Plan", because the Chinese government takes every five years as a cycle to make a comprehensive plan for national economic and social development. Once approved by the National People's Congress, this plan became the will of the entire country for five years. The completion and completion of any infrastructure project that can enter this planning directory must be a milestone, a historic event, not only with national but also international influence. The Hong Kong-Zhuhai-Macao Bridge is such an infrastructure project. Let us regard the Hong Kong-Zhuhai-Macao Bridge as an infrastructure project with global influence, which is a super project completed by the "infrastructure maniac" three years ago, and this scale of the project let us give it a code name - I.-E; let us regard Beijing Daxing International Airport as an infrastructure project with national influence, which is a sub-super project completed by the "infrastructure maniac" two years ago This scale of the project let us give it a code name - II.-E; let us see the Lanzhou-Chongqing railway from Lanzhou to Chongqing as an infrastructure project with inter-provincial influence, its story of opening the Flax Ridge Tunnel has touched the whole of China, this is a large project completed by the "infrastructure madman" four years ago, and this scale of infrastructure project let us give it a code name - III. -E; Let's regard the intelligent upgrading of the century-old port Qingdao Port as an infrastructure project with provincial influence, which is a medium-sized project completed by the "infrastructure maniac" a few years ago, and this scale of infrastructure project let us give it a code name - IV.-E Let's regard Xi'an Metro Line 9 as an infrastructure project with citywide influence, people from all over the world who come to Xi'an want to go to Lintong to see the Terracotta Warriors and Horses of Qin Shi Huang and Lishan Huaqingchi, there is a safe and convenient transportation option, this is a small project completed by the "infrastruc- 
ture maniac" last year, and this scale of infrastructure project let us give it a code name - V.-E. If the "infrastructure maniac" must have 1 I.-E completed every five years, 10 II.-Es will be completed at the same time, and 100 III.-- E was completed at the same time, and 1,000 IV.-E were completed at the same time, and finally 10,000 V.-E Completed at the same time. The quantitative relationship between the five types of infrastructure projects of different levels is an exponential relationship, which is the same as the "China Tower" model that describes China's territory. Chinese like to refer to the three infrastructure projects of railway, highway and airport civil aviation as "iron public aircraft", and its harmonic pronunciation is a figurative name - "iron rooster", "iron public aircraft" refers to all largescale infrastructure projects invested by the state and built by the state. During the 10th Five-Year Plan period (2001-2005), the total investment of the state in the "iron public machine" was 1.9 trillion yuan, the total investment of the 11th Five-Year Plan state in the "iron public machine" was 4.7 trillion yuan, and the total investment of the 12th Five-Year Plan countries in the "iron public machine" was 6.2 trillion yuan. The total investment of the 13th Five-Year Plan countries in the "iron public machine" is 15 trillion yuan, and the total investment of the 14th Five-Year Plan countries in the "iron public machine" is expected to exceed 35 trillion yuan.

\section{Irrational duration, a universal question with historical reasons}

An ancient country with three thousand years of farming culture and agricultural civilization, a proud and arrogant country that does not want to open its eyes to see the world for a hundred years, a country that misses the scientific revolution, the technological revolution, the industrial revolution and thus falls into a backward and beaten situation for a hundred years, a country that opens a new chapter with the raising of the five-star red flag is only 70 For many years, the country's ancient history has made its people proud, its modern history has humiliated its people, and its modern history and contemporary history have once again made its people stand up and become rich and strong. The pain of being humiliated by the Western powers one by one because of its low national strength and inability to protect itself is deep in my heart. To be backward is to be beaten-this is the first law of international politics recognized by New China and its people. In order to get rid of the passive situation of backwardness and beating as soon as possible, "how fast to save", "big dry and fast", "dora fast running", "great leap forward”, "leapfrog”... These irrational and passionate left-leaning slogans were once a powerful weapon for the first generation of leaders of New China to mobilize the masses of the people. During this period, although there were such and such mistakes, some of them even caused huge irreparable losses to the whole nation... However, the Chinese nation, which is good at correcting mistakes and drawing lessons, does not rigidly and dogmatically treat those mistakes and even mistakes, but draws lessons from them with a forward-looking and progressive attitude, but on the contrary holds an understanding attitude toward the historical reasons for the occurrence of those mistakes. Since the founding of the People's Republic of China, especially since the beginning of reform and opening up, China has made great strides forward, gradually and continuously shortening the gap with developed countries. It can now be said that in the history of human industrialization, the largest population, the greatest influence, and the largest scope of time and space are therefore an unprecedented industrialization process, which is being carried out in the ancient East on the land of China. China, which is in the historical process of large-scale industrialization, is simply a nationwide infrastructure construction site when viewed from a certain perspective. Every year, with the completion and commissioning of thousands of engineering projects, China continues to change its appearance and social productivity continues the way of the earth is changing, and the lives of ordinary people are constantly changing the content. The principles of Marx's political economy tell us that the development and emancipation of social productive forces is the fundamental driving force that causes changes in social, political, economic and cultural changes, and the reason for the great changes in China's economy and society is because the fundamental policies of reform and opening up, market economy, and scientific development, coupled with the extremely rare period of strategic opportunity, have liberated and developed the social productive capacity. If you look at it from the perspective of engineering construction and project management, the force unit that causes these changes is summarized in one word, and this keyword is construction; the technical unit that causes these changes is summarized in one word, and this keyword is engineering; the management unit that causes these changes is summarized in one word, and this keyword is the project. Engineering construction, project management, safety and quality, and construction cost, which were once professional terms in university classrooms, have now become common terms that can be used by ordinary people in Lujing. Looking at the history and reality of project management with Chinese characteristics, there is no doubt that there are three basic estimates. First, there is no doubt that engineering construction

Citation: Sun Jiajun. “The Universality, Major Harm and Deep Causes of Irrational Construction Periods". Medicon Engineering Themes 2.3 (2022): 49-56. 
and project management have made indelible contributions to China's economic and social development, and after more than 40 years of unremitting efforts in the way of catching up with the latecomers, the huge gap between China and developed countries at the beginning of reform and opening up has been greatly shortened Second, it is undeniable that there is still a considerable gap between the overall level of Science and Technology, the overall level of knowledge innovation, and the scientific level of engineering management in China compared with the developed countries in the world; third, it must be admitted that this gap is no longer the original gap at the beginning, but the gap that temporarily exists after the formation of a certain irreversible change trend between the latecomer and the first-mover A gap that does not widen the distance further is the kind of gap that can be gradually narrowed by relying on the gradual acceleration of the speed of the latecomer. How can this gap be further narrowed? It is related to the three fields of engineering innovation, technological innovation and basic science innovation in China A major scientific research topic. As far as engineering management is concerned, the view of this paper is that in order to narrow this gap, we must be good at summarizing and extracting operational knowledge from the experience of Chinese our own work, especially Chinese the major failures and major setbacks we have experienced. In addition, it is mathematically logically systematized into a theory that is easy for Chinese to grasp, which is a more practical innovation than the practice of "explaining China's engineering management problems according to the script and rigidly applying Western management theories".

The so-called irrational construction period refers to the in-depth and meticulous preliminary demonstration under the constraints of scientific procedures before the agreement between the two parties to the construction period A project is limited to how many years, months and days, the two parties to the agreed construction period do not have a number in mind, and both parties are unwilling to come up with an investment to ask a qualified third-party consulting company to make scientific calculations in advance of the construction period of the project, they take it for granted that the calculation of the construction period of a project is as purely in vain as calculating the fate of a person; the second is that the construction period is agreed upon by both parties Because the scientific data on the duration of the project was not obtained because sufficient preliminary demonstration was not made, subjective wishes, personal will, non-scientific factors, Non-engineering factors occupy the main aspects of the contradiction, and the role of decision-making tools such as operations research, duration quota, multi-objective comprehensive optimization, and ant colony intelligent algorithm for measuring the duration of the project has degenerated into a secondary aspect of the contradiction Third, in the process of implementation after the agreement between the two parties to the construction period, Party A inevitably emphasizes that the construction period is advanced, and Party B requires the extension of the construction period, resulting in a continuous change in the project duration. Excluding force majeure, specifically, the large advance of the project period is the first manifestation of the irrational construction period, and the serious lag of the engineering period is the second manifestation of the irrational construction period.

\section{Significant harm caused by irrational construction periods}

Irrational construction period is a common problem in project management.

If the irrational duration exists in I.-E, it will cause the greatest harm, and to some extent reflect some kind of mistake at the level of national decision-making.

If the irrational duration exists in II.-E, the harm it brings is significant, and it reflects a certain mistake at the decision-making level of a certain industry field in a certain degree of process.

If the irrational duration exists in III.-E, the harm it brings is relatively large, and to some extent reflects a certain mistake at the decision-making level in a certain professional field.

If the irrational duration exists in IV.-E, the harm it brings is intermediate, and to some extent reflects a mistake at the decision-making level in a certain technical field.

If the irrational duration exists in V.-E, the harm it brings is small, and to some extent reflects a certain mistake that often occurs at the level of a specific engineering decision.

Citation: Sun Jiajun. "The Universality, Major Harm and Deep Causes of Irrational Construction Periods". Medicon Engineering Themes 2.3 (2022): 49-56. 
Two cases in which irrational duration caused serious harm in I.-E

The industrial fairy tale of the whole people smelting steel

In the depths of the Chinese national memory, there is an "engineering project" called large-scale steelmaking, which requires that the output of steel be doubled from the original 5.35 million tons to 10.7 million tons in a one-year construction period On December 19,1958 , it was announced that the task of doubling steel production was completed 12 days ahead of schedule, with steel production of 11.08 million tons and pig iron production of 13.69 million tons. In fact, only 8 million tons of qualified steel are available, and the 3 million tons of soil steel and 4.16 million tons of earth and iron smelted cannot be used at all, and the direct economic loss is about 20 billion yuan [1].

How high are those money yards

Start, glide, the nose up, straight into the sky.

On the afternoon of May 5, 2017, Shanghai Pudong International Airport, the first trunk aircraft C919 with independent intellectual property rights in China with international mainstream standards, successfully made its first flight. In the crowd cheering and celebrating, 87-year-old Cheng, the deputy chief designer of Yunshi, came to witness this moment from time to time.

\section{From time to time, Mr. Cheng said}

This is a very important moment in history, a major step, and it will be a very strong stroke in the history of our country's aviation development. In order for China to buy foreign aircraft, we have to sell hundreds of millions of shirts to exchange for an airplane, and we Chinese, will it always be like this on this low end? The money we bought the plane, some people have done calculations, with a brand new denomination of the highest hundred yuan banknote piled up, has been higher than the Shanghai Jin Mao Tower, not only Jin Mao Tower, higher than the ten Jin Mao Tower, not only ten, higher than a hundred Jin Mao Tower, has reached into space, like Sun Monkey's golden hoop stick, stretched into space, such a money pillar cut down a section, When it is used in China, you see how much culture it will bring about, the rise of technology, and the renewal of industrial industries.

For this passage of Elder Cheng's words, we should further interpret the arithmetic aspect so that people can understand it more deeply. After Yun ten was laid off, the money that Civil Aviation of China bought Boeing and bought Airbus would be a high pillar of money if it could rise on the ground?

Yun Ten was laid off in 1986. The oral theory of the dismount is that shipbuilding is not as good as renting a ship, and renting a ship is not as good as buying a ship. Behind this oral theory is actually a plan to go bankrupt - to exchange the market for leapfrog progress in core technology. Facts have proved that the core technology that maintains the core competitiveness of a country's survival can only be achieved by relying on the self-reliance of its own nation and accumulating little by little, and there is no such model as so-called leapfrog progress. In the 30 years from 1987 to 2017, Aviation of China purchased no less than 3,500 passenger aircraft of various types, of which Boeing did not less than 1,000 No less than 1,000 aircraft and Airbus. In terms of buying Boeing and buying Airbus alone, the total cost is not less than 800 billion yuan. The 100 yuan denomination is 10,000 yuan a wad, and its thickness is about 2.5 $\mathrm{cm}$. What a pillar of money would be if 800 billion yuan was raised on the ground?

Ten thousand yuan - 2.5 centimeters

400,000 yuan -1 meter

Four million yuan - 10 meters

Forty million yuan - 100 meters

400 million yuan - $1 \mathrm{~km}$

400 billion yuan - 1,000 kilometers

800 billion yuan - 2,000 kilometers

Citation: Sun Jiajun. "The Universality, Major Harm and Deep Causes of Irrational Construction Periods". Medicon Engineering Themes 2.3 (2022): 49-56. 


\section{Two kinds of harm caused by irrational construction periods in V.-E}

Behind the irrational construction period in V.-E, there are two kinds of hazards: one is to regard the advance of the construction period as an important performance of project management to promote the "short-lived project"; the other is to delay the elimination of the construction period, resulting in the binding force of the contract duration.

\section{Performance Chemical Period Advance Boost "Short-lived Project"}

Treating the advance of the construction period as an important performance of project management to promote the "short-lived project" is a hazard of the irrational construction period. Over the past few years, the news reports in advance of the performance chemical period have been incessant, the project reports in advance of the performance chemical period have been heard endlessly, the leaders of the performance chemical period have spoken endlessly, how many days ahead of time a certain project has been completed, a certain project has been completed several months ahead of schedule, and the completion of a certain project has become a customary phrase for the media to publicize and report on the completed project, such as the advance of the Qinghai-Tibet Railway 1 year, the Beijing-Shanghai high-speed railway was put into trial operation 2 years in advance, which was once used as the entry point of the great cause to be publicized and amplified, making it easy for people to have the illusion that the larger the advance of the construction period, the greater the project performance. What does it mean to advance the schedule? It's meaningful. For the people, it is possible to open the traffic in advance, check in early, prevent flood control and generate electricity in advance, and generate economic benefits in advance; for owners and contractors, it is to refresh records, create miracles, and win fame, of course, everyone is happy. But the other side of the matter is those "short-lived projects" that make people frightened. It is not difficult to find that those "short-lived projects" have a black hand behind the scenes, that is, to catch up with the irrational construction period under the guidance of concepts.

The delay in the chemical period of the elimination of the item leads to the binding force of the contract duration if there is no

The delay in the elimination of the construction period leads to the contract duration if there is no, which is another hazard of the irrational construction period. The contract duration refers to the construction period confirmed in the contracting contract by the contracting parties under the guidance of the fixed construction period according to the specific conditions of the project construction, after bidding and bidding or consensus. Once the contract period is signed, it has a mandatory binding effect on both parties to the contract and is protected and restricted by the national economic contract law. However, in the actual implementation, the contract construction period lags behind has become a common thing, and very few are strictly implemented according to the contract duration, which is particularly prominent in small and medium-sized engineering projects. Once the contract period is delayed, the construction management parties first attribute the problem to the insufficient equipment of the construction unit and the poor construction organization [2]. And the construction unit will find out many problems of the construction unit, such as the fact that the preparation of the construction site does not meet the requirements of the contract, the funds are slow to arrive, and the design changes are large, so the big things are small and the small things are small, and finally the result of no claim and no punishment is reached.

\section{Deep reasons why irrational durations exist \\ Subjective desire ignores objective laws as the first reason}

Every project to be built, every project to be built, no matter what kind of specific functions the construction unit gives it, in terms of the operation process of its transformation from paper engineering to real engineering, it plays a substantial role in what is called man-machine-environment system engineering. Based on this, each project can be defined as a large-scale human-machine-environment system engineering, which relies on the optimization of the configuration of the three elements of the human-machine-environment to achieve its overall function. In 1981, under the personal guidance of the famous scientist Academician Qian Xuesen, a comprehensive fringe technology science - human-machine-environment system engineering was born in China. Man-Machine-Environment System Engineering (MMESE) is the application of systems science theory and systems engineering methods. Correctly handle the relationship between the three major elements of the human-machine environment, and deeply study the optimal combination of hu- 
man-machine-environment systems [3]. From this theory, we can find out what objective laws are contained in a project or a project. Any project is inseparable from environmental factors, and as far as the environment is concerned, it is subject to the laws of nature; any project is inseparable from man's purposeful activities, and in terms of man's purposeful activities, it is subject to economic laws; any project must have special instruments, tools, machines, and equipment as means, and as far as machine tools are concerned, it is subject to the law of technological evolution. As a whole formed by the three factors of man-machine-environment, that is, the project itself, it must be subject to the joint superposition of these three laws, and the engineering duration as an intrinsic factor of the project must also be subject to the joint superposition of these three laws. The irrational construction period is fundamentally the failure to recognize that the engineering period also has its own objective laws.

\section{The will of the Chief in place of the scientific process is the second reason}

The duration of the project is not a simple mathematical problem similar to the established distance and the time of the established speed, but a complex problem that can only be calculated and solved by using operations research as a tool and the support of computer and network technology. This is because of this duration, cost, safety and quality4Factors, they every 2 There is a complex nonlinear relationship between them, and they are every3There is a more complex nonlinear relationship between them 4 There is an extreme complexity between them Nonlinear relationships. The basic mathematical method of dealing with these complex relationships is called multi-objective integrated optimization, and specialized computing techniques are called ant colony algorithms (ant colony optimization, ACO) [4]. Every owner wants their project to be short, of good quality, economical and safe, but the key question is how to do so. Systems Engineering Proto Theory tells us that the construction period must take into account the cost, quality and safety, and under the condition of taking into account, there must be a numerical range of the minimum construction period that cannot be shorter than a number of days, and the longest cannot be longer than a number of days. Restrictions are prescribed The Values window for a reasonable duration. Engineering The actual construction period, only by falling into this window can we find the whole project functional optimize. This is Project management progress manager Objective laws that must be observed. Respect engineering the objective law of the construction period, Acknowledging the incompressibility of the duration of the project under the premise of various constraints, according to the principle of process control of progress Strictly step by step to implement, engineering safe Quality and Comprehensive benefits, it will naturally arise from this foundation. China The first bridge ever built over the Yellow River was built in Lanzhou city, known as the Yellow River Iron Bridge, The Americans were the architects of the bridge, and the Germans were the engineers who built it the bridge 1907 Year survey and design, 1908 Construction began in the spring August 19, 1909 Day completion pass until August 19, 1989 The bridge was in full service on the day80Years later, the German company that built it, Tai lai \& Co., remembers it. Someone from Germany came to Lanzhou to handle the retirement procedures for the bridge. The Lanzhou Yellow River Iron Bridge, its quality, its lifespan, its perfection and the professionalism of the German engineers who built the bridge shocked everyone who knew the history of the bridge [5].

\section{Under the buyer's market pattern, Party A's strength and Party B's weakness are the third reason}

After more than 40 years of unremitting efforts, the economic form of Chinese society has undergone earth-shaking and tremendous changes, and the kind of products and materials at the beginning of reform and opening up more than 40 years ago are extremely scarce. The backward situation in which cotton coupons, cloth, oil, food stamps, and food stamps are more effective than currencies has become a legendary history, and the market pattern has changed from the original severely deformed seller's market The current buyer's market is rich in materials and rich in goods, and on the whole, this is a great achievement of social and economic progress to promote the progress of social life. However, everything has positive and negative aspects, the simple buyer's market also has its drawbacks, take engineering construction, project management, resulting in an imbalance in the economic relationship between A and B, specifically that Party A is always in a strong position and Party B is always in a weak position. Specific to the project duration of the matter, under the normal relationship conditions of Party A and Party B, Party B should also have a considerable right to speak, negotiate and dispose of the construction period, but the reality is not so, the construction period is often something that Party A can decide, and Party B can only be duplicitous about the construction period agreement in order to have work to survive.

Citation: Sun Jiajun. “The Universality, Major Harm and Deep Causes of Irrational Construction Periods". Medicon Engineering Themes 2.3 (2022): 49-56. 


\section{Conclusions}

The project duration is not only the hub of the project intrinsically related factors, but also the hub of the external related factors of the project, and it is also the sensitive nerve-intensive hub of the interested parties in the project, which is a focal issue in the project management. Rome was not built in a day, the Long March was not completed in one step, and the construction period of the project had its own objective laws. Respecting the objective law of the project duration, consciously using this law to serve the project, and acknowledging that the project duration is incompressible under the premise of various constraints, is the theoretical cultivation that every project manager should have. It is an extremely wrong concept to regard the project duration as a "sponge that still has gaps at the disposal of others", and the irrational construction period under the guidance of this concept is a universal problem in project management.

\section{References}

1. Baidu Encyclopedia entry big smelting steel.

2. Ma Yuzhen. "Causes and Countermeasures of Contract Delay". Inner Mongolia Water Conservancy 3 (2008).

3. Long Shengzhao. "The theory of man-machine-environment system engineering and its significance in the development of productive forces". The source of literature is "Research Progress of Man-Machine-Environment System Engineering (Volume I)" Science and Technology Press (1993).

4. Liu Yuan. "Duration-Cost-Quality Multi-objective Optimization of Engineering Projects Based on Multiple Colony Colony Algorithms and Its Application Research". Jiangsu University of Science and Technology, Master's Theses (2012).

5. Chen Qiwen. Who Can Rewrite History Xinhua Digest 7 (2013): 82-85.

Volume 2 Issue 3 March 2022

(C) All rights are reserved by Sun Jiajun. 